Nachruf

Z. Epileptol. 2019 · 32:244-247

https://doi.org/10.1007/s10309-019-0277-3

Online publiziert: 24. Juli 2019

(c) Springer Medizin Verlag GmbH, ein Teil von Springer Nature 2019

Mit Frederick (Fred) Andermann ist einer der ganz großen klinischen Epileptologen der zweiten Hälfte des 20. und frühen 21. Jahrhunderts im Alter von 88 Jahren gestorben. Neben seinem syndromatologischen und genetisch orientierten Schwerpunkt war er u.a. auch wegweisend in der Etablierung der Epilepsiechirurgie bei Kindern und Erwachsenen. Fred war nicht nur physisch eine stattliche Erscheinung, sondern auch ein sehr angenehmer, großzügiger, offen- und warmherziger Mensch. Mit ihm konnte man lange und geistreiche Diskussionen auch außerhalb der Epileptologie führen; er war in seiner Argumentation stets klar und präzise, aber nie aggressiv oder gar verletzend. Als ein Kollege in Montreal 1993 über den gerade in die Kinos gekommenen Spielfilm „Schindlers Liste“ von Stephen Spielberg lästerte, sagte er nur: „Ich denke, es ist ein guter Film“.

Fred Andermann wurde am 26.09.1930 in Czernowitz im damaligen Königreich Rumänien (bis 1918 als Provinz Bukowina - deutsch: Buchenland - der östliche Zipfel der österreichisch-ungarischen Monarchie; heute: Chernivtsi, Ukraine) in eine wohlhabende jüdische Kaufleutefamilie geboren. Als die Rote Armee 1940 dieses Gebiet der Sowjetunion anschloss, musste die Familie ihre Heimat innerhalb von $24 \mathrm{~h}$ verlassen und floh zunächst nach Bukarest, wo die Mutter in einer Organisation zur Rettung von Kriegswaisen mitarbeitete. Dadurch entging die Familie der Deportation nach Sibirien. Viele der in den später von deutschen Truppen besetzten Gebieten verbliebenen Familienmitglieder wurden im Holocaust von den Nazis ermordet. Nach dem 2. Weltkrieg zog die Familie nach Wien und Paris, be-

\section{Günter Krämer}

Neurologie FMH, Neurozentrum Bellevue, Zürich, Schweiz

\section{In memoriam Frederick („,Fred $\left.{ }^{\prime \prime}\right)$ Andermann (1930-2019)}

vor sie 1950 nach Kanada auswanderte. Fred gehörte damit zur „zweiten Welle“ von mit ihren Eltern eingewanderten europäischen Emigranten mit dem Vorteil einer medizinischen Sozialisation in Nordamerika [1-4].

Fred Andermann erwarb 1952 zunächst einen Bachelor of Science(B.Sc.)Titel an der Montrealer McGill-Universität, danach begann er an der gerade neu gegründeten frankophonen Université de Montréal sein Medizinstudium. Gleichzeitig war er noch für das Familienunternehmen „Anderco Import“ tätig, reiste durch Kanada und verkaufte Kämme, Scheren und Musikinstrumente. Im Jahr 1957 schloss er das Medizinstudium mit dem M.D. ab.

Nach dem Studium absolvierte er seine Facharztweiterbildung am Montreal Neurological Institute und Hospital („Neuro“), wo er für seine gesamte Kar- riere verblieb. Als Assistenzarzt war er besonders in den akademischen Lehrkrankenhäusern beschäftigt, wie dem „Hôtel Dieu“ oder dem Krankenhaus „Notre Dame“. Für die fachspezifische Weiterbildung war zunächst der Neurochirurg und Epileptologe Wilder Penfield (1891-1976) zuständig, nach dessen Tod der Neurochirurg und Epileptologe Theodore Rasmussen (1910-2002), der das von Penfield begonnene Montrealer Epilepsieprogramm fortsetzte und weiterentwickelte [4].

Fred hatte an der McGill-Universität in Montreal Professuren in den Abteilungen für Neurologie, Neurochirurgie und Pädiatrie inne und war für 35 Jahre Direktor des Epilepsiebereichs und der Epilepsieambulanz am Montreal Neurological Hospital und Institut. Im Jahr 2014 zog er sich aus der klinischen Arbeit und der Ausbildung von Fellows zurück, reiste je-

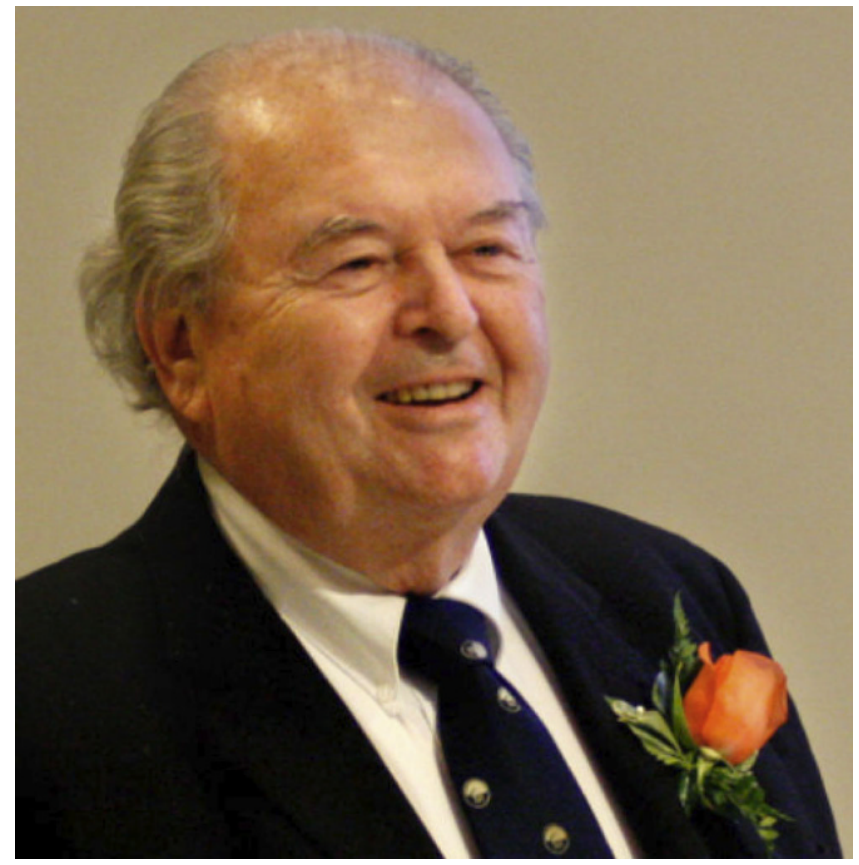

Abb. $1<$ Fred Andermann 


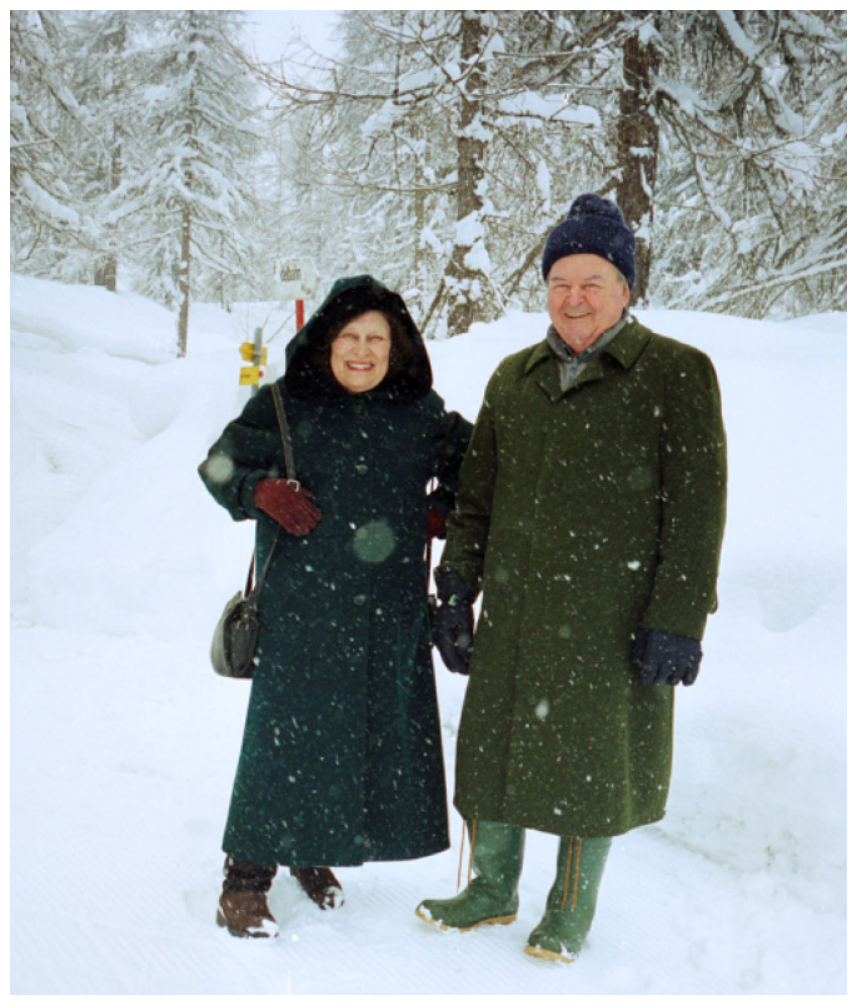

Abb. $2<$ Eva und Fred Andermann 2001 in Sils Maria

doch weiterhin zu medizinischen Konferenzen und veröffentlichte wissenschaftliche Artikel.

Seine erste von insgesamt über 500 PubMed-gelisteten Publikationen war eine 1959 in Neurology erschienene Kasuistik von 2 Patienten mit paroxysmaler Dysarthrie und Ataxie bei multipler Sklerose, seine letzte erschien im Januar 2019 als Koautor einer federführend von Sam Berkovic verfassten Arbeit in Brain zur Molekulargenetik, Klinik und Pathologie der Kufs-Krankheit. Bei Research Gate hatte er Ende Juni 2019 beeindruckende 36.161 Zitierungen [5] und er war Mitherausgeber von 25 Büchern mit einem weit gesteckten Themenbereich u.a. von "Migraine and Epilepsy“ (1987) über „Chronic Encephalitis and Epilepsy. Rasmussen's Syndrome“ (1991), „Magnetic Resonance Scanning and Epilepsy“ (1994), „Dysplasias of Cerebral Cortex and Epilepsy“ (1996), ,Reflex Epilepsies and Reflex Seizures“ (1998), „Epilepsy and Movement Disorders“ (2002) bis zu „The Causes of Epilepsy. Common and Uncommon Causes in Adults and Children“ (2011).

Fred Andermann hatte ein besonderes Talent darin, ein multidisziplinäres
Team um sich herum aufzubauen und mit diesem wissenschaftliche Studien durchzuführen. Dabei spielten Fellows aus 15 verschiedenen Ländern eine wichtige Rolle. Stellvertretend seien hier Bassel W. Abou-Khalil (Libanon/USA), Sam Berkovic (Australien; auch bei Eva Andermann), Fernando Cendes (Brasilien), Antonio Gambardella (Italien), Yvonne Hart (England), Heinz-Joachim Meencke (Deutschland), André Palmini (Brasilien), Hermann Stefan (Deutschland), Pierre Thomas (Frankreich) und Eugen Trinka (Österreich) genannt.

Nachdem er u.a. von 1993 bis 1997 Vorsitzender der „Task Force on Classification" der ILAE und danach weiterhin Mitglied dieser Task Force war, hatte er sich aus der in Anbetracht der neuen ILAE-Klassifikationen von 2017 und Gegenvorschlägen hauptsächlich von der Gruppe um Hans Lüders wieder sehr aktuellen Diskussion etwas ernüchtert zurückgezogen.

Er war weltweit ein sehr häufig eingeladener Gastredner auf Kongressen und Symposien sowohl auf internationaler oder nationaler Ebene als auch bei fokussierten Symposien oder Workshops. Ich hatte die Freude, Fred und
Eva $2001 \mathrm{zu}$ einem gemeinsam mit Hermann Stefan organisierten Workshop zur Lateralisation und Lokalisation von Anfallssymptomen in die Schweiz einladen zu können. Dieser fand in einer kleinen Villa oberhalb des legendären Hotels „Waldhaus“ in dem Dorf Sils Maria unweit von Sankt Moritz im Oberengadin statt. Beide haben mir später wiederholt versichert, dass sie diese Veranstaltung besonders genossen haben (• Abb. 2). Die von den Nazis 1944 in ihrem holländischen Exil verhaftete, in ein $\mathrm{KZ}$ verbrachte und kurz vor Kriegsende an Typhus verstorbene jüdische Schülerin Anne Frank, die durch ihr Tagebuch weltbekannt wurde, verbrachte 1935 und 1936 ihre Sommerferien mit ihrer Pariser Tante genau in diesem Haus [6].

Fred Andermann hat sein gesamtes akademisches und wissenschaftliches Leben in Montreal verbracht, war aber ursprünglich ein polyglotter Europäer, der sich mit seinen Patienten u.a. auch in Deutsch, Französisch, Italienisch, Rumänisch oder Russisch unterhalten konnte. Er machte keinen Unterschied zwischen Nationalitäten oder Arm und Reich. Beim Kontakt mit seinen Patienten begab er sich bei Bedarf nicht nur in sprachlicher Hinsicht auf deren Ebene (• Abb. 3).

Sein Lieblingsbuch waren die 1953 erschienenen „Maghrebinische Geschichten" von Gregor von Rezzori (1914-1998), der wie er in Czernowitz geboren wurde. Das Buch handelt von dem Phantasieland Maghrebinien - ein wenig Österreich, etwas Balkan, ein bisschen Orient - in dem seinen Figuren wundersame, pfiffige und heitere Erlebnisse widerfahren. Sein Lieblingsautor war der deutsche Dichter, Schriftsteller und Journalist Heinrich Heine (1797-1856), der zugleich als „letzter Dichter der Romantik" als auch deren Überwinder gilt. Fred liebte die Natur und Tiere (er hatte außerhalb von Montreal eine Farm, wo er viele Wochenenden verbrachte), am wichtigsten war ihm aber seine große Familie mit den 3 Kindern Lisa, Anne und Mark sowie den 6 Enkelkindern Ben, Hannah, James, Kaija, Lara und Leila [1].

Andermann war u.a. zusammen mit Juhn Wada 1977 Gründungsmitglied, 


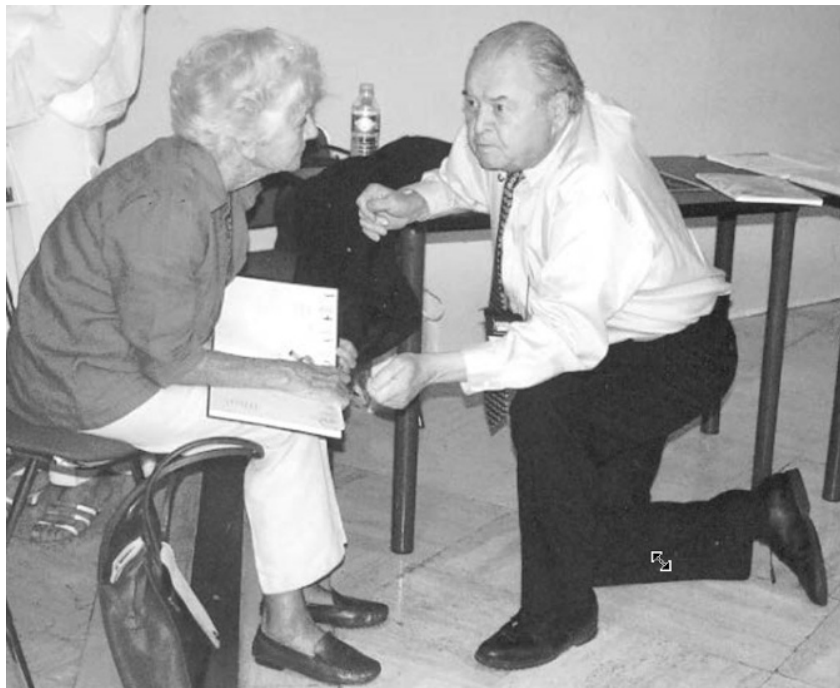

Abb. $3<$ Fred Andermann im Gespräch

1985 bis 1987 Präsident und 1987 bis 1989 Past-Präsident der Kanadischen Liga gegen Epilepsie, darüber hinaus auch Präsident der Kanadischen Neurologischen Gesellschaft, der Kanadischen Gesellschaft für Klinische Neurophysiologie, der Kanadischen Vereinigung von Kinderneurologen und der „Eastern EEG Society“. Von 2001 bis 2005 war er alleiniger Vizepräsident und von 2005 bis 2009 2. Vizepräsident der ILAE.

Die Liste seiner akademischen Auszeichnungen ist beeindruckend und beinhaltet u.a.:

- 1989 „Ambassador for Epilepsy“ durch die ILAE und das IBE,

- 1995 Forschungsanerkennungspreis der American Epilepsy Society (AES) und Milken-Familienstiftung,

- 1999 Wilder-Penfield-Award der Kanadischen Liga gegen Epilepsie,

- 2000 Lennox-Award der AES,

- 2003 Prix Wilder Penfield der Provinz Quebec,

- 2004 Alan Ross Award von der Canadian Pediatric Society,

- 2006 Neuro Lifetime Achievement Award des Montreal Neurological Institute (MNI) und Hospital,

- 2006 Officer of the Order of Canada der Kanadischen Regierung

- 2007 Bernard Sachs Distinguished Award for Research der US-amerikanischen Child Neurology Society,

- 2007 Ehrenpreis der Gesellschaft für Neuropädiatrie (vormals Peter-EmilBecker-Preis),
- 2011 Fellow der Royal Society of Canada,

- 2013 Order of Québec und

- 2015 „Lifetime Achievement Award“ durch die ILAE und das IBE.

Im Jahr 1965 heiratete Fred seine Frau Eva, geborene Deutsch, die er 1958 als Studentin an der McGill-Universität kennengelernt hatte. Sie stammte aus einer jüdischen Familie in Polen und hatte ein ähnliches Schicksal hinter sich wie er. Mehrere Familienmitglieder wurden zu Beginn des Polenfeldzuges von den Nazis ermordet, ihr selbst gelang mit ihren Eltern zunächst die Flucht in die Ukraine, wo sie nach der deutschen Besetzung nur überlebten, weil die Eltern als jüdische Ärzte zur Seuchenbekämpfung in den Dörfern eingesetzt und später von polnischen Landsleuten versteckt worden waren. Nach Kriegsende übersiedelte die Familie nach Montreal. Evas Mutter, Mina Deutsch, hat über diese Erlebnisse ein u.a. auch ins Deutsche übersetztes, sehr lesenswertes autobiografisches Buch geschrieben [7].

Eva wurde ebenfalls Neurologin und Epileptologin mit einem genetischen Schwerpunkt. Sie war langjährige Leiterin der Neurogenetischen Einheit am Montreal Neurological Hospital and Institute. Im Jahr 1972 beschrieben Fred und Eva federführend erstmals die hereditäre motorisch-sensible Neuropathie mit Agenesie des Corpus callosum (OMIM \#218000) und 1983 erstmals das Aktionsmyoklonien-progrediente Nieren-
versagen-Syndrom (OMIM \#254900), die auch als Andermann-Syndrom I und Andermann-Syndrom II bezeichnet werden. Von ihren Kindern haben Lisa und Mark die berufliche Orientierung der Eltern teilweise übernommen, und Lisa hat u. a. 2008 mit Stephen Schachter (Boston) den Band „Stories of Living with Seizures from around the Globe“ in der Reihe „Epilepsy in Our World“ herausgegeben.

In einem Buch zur Geschichte der Neurologie in Quebec von 2011 heißt es u.a. „Bis vor kurzem leitete Dr. Andermann die Abteilung für Epilepsie am MNI und setzte mit seiner Frau Dr. Eva Andermann seine Studien zu den Mechanismen der Epilepsie fort, die vor einigen Jahrzehnten begonnen worden waren.... Ihre gemeinsame Arbeit, die klinische und genetische Forschung kombiniert, hat das Wissen über die Klassifizierung, Diagnose und Behandlung von Epilepsie erweitert ..." [8].

In seinen letzten Lebensjahren hatte Fred mit zunehmenden gesundheitlichen Problemen zu kämpfen. Am 16.06.2019 starb er in Montreal.

\section{Korrespondenzadresse}

\section{Dr. med. Günter Krämer}

Neurologie FMH, Neurozentrum Bellevue Theaterstr. 8, 8001 Zürich, Schweiz g.kraemer@epilepsie-med.de

Danksagung. Ich danke Hans Holthausen und Eugen Trinka für wertvolle Hinweise.

\section{Literatur}

1. International League Against Epilepsy (2019) Dr. Frederick Andermann, OC, OQ, MD, FRCPC. September 26, 1930 - June 16,2019. https://www.ilae. org/files/dmfile/Andermann-Frederick-20190616.pdf.Zugegriffen: 30. Juni 2019

2. Krämer G (2012) Lexikon der Epileptologie. Hippocampus, Bad Honnef, $\mathrm{S} 61$

3. Holthausen H (2007) Peter-Emil-Becker-Preis für Eva und Fred Andermann. Mitt Ges Neuropädiatr 2:3-4

4. Stahnisch FW (2008) Zur Zwangsemigration deutschsprachiger Neurowissenschaftler nach Nordamerika. Der historische Fall des Montreal Neurological Institute. In: Keil G, Holdorff B (Hrsg) Schriftenreihe der Deutschen Gesellschaft für Geschichte der Nervenheilkunde, Bd. 14. Königshausen \& Neumann, Würzburg, S440-472

5. ResearchGate (2019) Frederick Andermann. https://www.researchgate.net/profile/Frederick Andermann;. Zugegriffen:30. Juni 2019 
6. Ohler N (2014) Die Abgründe von Sils-Maria. https://www.zeit.de/2014/52/juedischegeschichte-sils-maria-schweiz;. Zugegriffen: 30.

Juni 2019

7. Deutsch M (1994) Mina's Story: A Doctor's

Memoir of the Holocaust. ECW, Toronto (deutsche

Ausgabe: Deutsch M (2013) Minas Geschichte.

Die Erinnerungen einer Ärztin an den Holocaust

(„Jüdische Memoiren“, Band 20). Hentrich \&

Hentrich, Berlin)

8. Goulet D (2011) Histoire de la neurologie au

Québec. Carte Blanche, Outremont, S326-328

Hier steht eine Anzeige.

算 Springer 